\title{
Quantifying the refractive index dispersion of a pigmented biological tissue using Jamin-Lebedeff interference microscopy
}

\author{
Doekele G Stavenga, Hein L Leertouwer and Bodo D Wilts
}

Jamin-Lebedeff polarizing interference microscopy is a classical method for determining the refractive index and thickness of transparent tissues. Here, we extend the application of this method to pigmented, absorbing biological tissues, based on a theoretical derivation using Jones calculus. This novel method is applied to the wings of the American Rubyspot damselfly, Hetaerina americana. The membranes in the red-colored parts of the damselfly's wings, with a thickness of $\sim 2.5 \mu \mathrm{m}$, contain a pigment with maximal absorption at $\sim 490 \mathrm{~nm}$ and a peak absorbance coefficient of $\sim 0.7 \mu \mathrm{m}^{-1}$. The high pigment density causes a considerable and anomalous dispersion of the refractive index. This result can be quantitatively understood from the pigment absorbance spectrum by applying the Kramers-Kronig dispersion relations. Measurements of the spectral dependence of the refractive index and the absorption are valuable for gaining quantitative insight into how the material properties of animal tissues influence coloration.

Light: Science \& Applications (2013) 2, e100; doi:10.1038/lsa.2013.56; published online 27 September 2013

Keywords: absorption; chitin; insect wings; Jones calculus; Kramers-Kronig relations

\section{INTRODUCTION}

Pigmentary coloration occurs in tissues in which pigments selectively absorb light in a certain wavelength range while light outside this absorption band is scattered, thus coloring the tissue. Pigmentary coloration is common in many animals; for instance, blue-absorbing carotenoids produce the yellow color of the feathers of many songbirds, ${ }^{1,2}$ and the ultraviolet- and blue-absorbing pterins in pierid butterfly wing scales produce white (Small White, Pieris rapae) and yellow (Eastern Pale Clouded Yellow, Colias erate) wings, respectively. ${ }^{3}$ Due to their usually high concentration, the pigments act as effective highpass spectral filters. However, because absorption is intimately linked to the refractive index, a strongly absorbing pigment can increase the refractive index and can thus strongly influence the coloration.

A quantitative understanding of the coloration of a tissue requires an analysis of both the spatial distribution and the optical properties of the tissue components. For anatomy, well-established methods include light and electron microscopy, and for pigment characterization, several spectrophotometry approaches exist. ${ }^{4}$ For microscopic objects, refractive index matching fluids are often used, with JaminLebedeff interference microscopy being one of the most sensitive methods. Thus far, such investigations have been conducted only on transparent, unpigmented biological tissues. ${ }^{5-7}$ In the visible wavelength range, transparent materials have a fairly constant refractive index, i.e., they exhibit little dispersion; thus, refractive index measurements are commonly restricted to one wavelength. However,
Jamin-Lebedeff interference microscopy is also suitable for a broad wavelength range, as we recently demonstrated using bird feathers and butterfly wing scales, where we determined the spectral dependence of the refractive index of the main components, keratin and chitin. ${ }^{8}$ Here, we show that the Jamin-Lebedeff interference microscopy method can also be applied to pigmented tissues, where the dispersion is not insignificant. We first present a theoretical derivation for the measurements, and we subsequently demonstrate the method using the densely pigmented, red wing regions of the American Rubyspot, Hetaerina americana, a damselfly found throughout North America.

\section{MATERIALS AND METHODS}

Animals

Specimens of the American Rubyspot, Hetaerina americana, were obtained from the National Museum of Natural History (Naturalis, Leiden, The Netherlands; curator Dr J van Tol). Small sections were cut from the wings for Jamin-Lebedeff interference microscopy and microspectrophotometry.

Scanning electron microscopy

The ultrastructure of the wing pieces was investigated with an XL-30 ESEM scanning electron microscope (Philips, Eindhoven, The Netherlands). Prior to imaging, the wing pieces were sputtered with palladium.

Computational Physics, Zernike Institute for Advanced Materials, University of Groningen, NL-9747 AG Groningen, The Netherlands

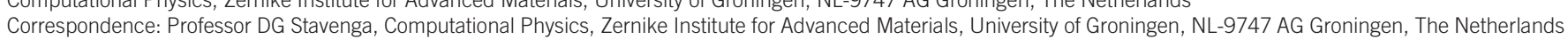
E-mail: D.G.Stavenga@rug.nl

Received 25 March 2013; revised 28 May 2013; accepted 30 May 2013 


\section{Spectrophotometry}

Reflectance spectra of the differently colored wing areas were measured in air with a bifurcated fiber-optic probe. The instrument comprises six light guides, delivering light from a halogen-deuterium source (AvaLight-D(H)-S; Avantes, Eerbeek, The Netherlands). These illuminating light guides collectively surround a central fiber that acts as a collector of scattered light (spot diameter: $\sim 1 \mathrm{~mm}$ ), which is delivered to a fiber optic spectrometer (Avantes, AvaSpec2048). A white diffusing reflectance standard (Avantes WS-2) was utilized as the reference. Using the oscillations in the reflectance spectra, the average wing membrane thickness was derived via a thin film analysis as presented previously. ${ }^{9}$ Absorbance spectra of the membranes in between the wing veins were measured with a microspectrophotometer (MSP), which consisted of a xenon light source, a Leitz Ortholux microscope and a spectrometer. The microscope objective was an Olympus $20 \times$, NA 0.46 . The measurements were performed on wing pieces immersed in a refractive index matching fluid to reduce scattering artifacts. Due to the optics of the glass, the spectral range of the MSP is limited to wavelengths greater than $380 \mathrm{~nm}$. For the Kramers-Kronig calculations, the absorbance coefficient spectrum was extrapolated by assuming that the spectrum consists of two absorption bands, $\alpha$ and $\beta$, which can be described by the modified lognormal function: $\kappa=a \exp \left[-b x^{2}\left(1+c x+d x^{2}\right)\right]$, with $d=3 c^{2} / 8$ and $x=\log _{10}\left(\lambda / \lambda_{\text {max }}\right)$ (see Ref. 10). For the $\alpha$ band in Figure $7 \mathrm{a}$, the parameters were $a=0.374 \mu \mathrm{m}^{-1}, b=215, c=7.07$, and $\lambda_{\max }=491 \mathrm{~nm}$, and for the $\beta$ band, $a=0.598 \mu \mathrm{m}^{-1}, b=476, c=-11.0$ and $\lambda_{\max }=300 \mathrm{~nm}$.

\section{Jamin-Lebedeff interference microscopy}

The wing pieces were placed on a microscope slide and covered by a cover slip after being immersed in a fluid with a refractive index varying between 1.46 and 1.64 (series A of Cargille Labs, Cedar Grove, NJ, USA). The wavelength dependence of the refractive index of each immersion fluid was derived from the refractive index value given for a wavelength of $586 \mathrm{~nm}$ and from its Abbe number by using the Cauchy equation $n(\lambda)=A+B / \lambda^{2}$ and by calculating the parameters $A$ and $B$. The microscope slide was mounted on the stage of a Zeiss Universal Microscope set up for Jamin-Lebedeff polarizing interference microscopy. The microscope objective was a Zeiss Pol-Int I 10×/0.22. As a light source, we used a halogen lamp, and specific wavelengths were selected by inserting narrowband interference filters (halfwidth: $\sim 15 \mathrm{~nm}$ ) below the condenser. At each wavelength, photographs were taken at a series of angular positions of the analyzer with a Coolsnap ES monochrome camera (Photometrics, Tucson, AZ, USA). The images were subsequently evaluated with Image $J{ }^{11}$ and the obtained sinusoidal intensity profiles were analyzed with Matlab routines.

\section{RESULTS AND DISCUSSION}

\section{Theory of Jamin-Lebedeff interference microscopy for absorbing media}

To determine the refractive index of an object with Jamin-Lebedeff interference microscopy, the object is embedded in a reference fluid with a known refractive index. Figure 1 presents a diagram of the system. ${ }^{5,6}$ A light beam delivered via the condenser of the microscope first passes through a linear polarizer, P. A birefringent beam splitter, BS, divides the beam into an ordinary and extraordinary ray. The polarization is then reversed by a half-wave plate, $\mathrm{H}$. One ray proceeds unhindered through the immersion fluid (Figure 1, grey), while the other ray passes through the object $\mathrm{O}$. The two rays are subsequently combined by a beam combiner, $\mathrm{BC}$. The joined rays then pass through

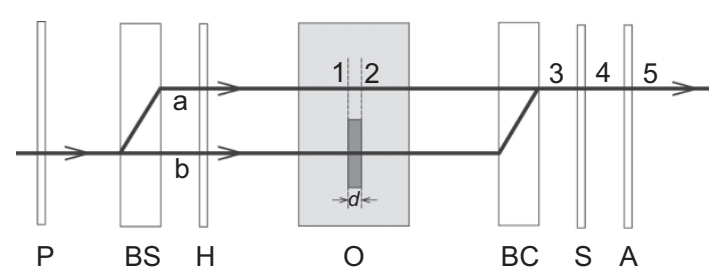

Figure 1 Diagram of the Jamin-Lebedeff interference microscope. P, polarizer; BS, beam splitter; $\mathrm{H}$, half-wave plate; $\mathrm{O}$, object; $\mathrm{BC}$, beam combiner; $\mathrm{S}$, Sénarmont compensator; $A$, analyzer. An object with thickness $d$ is immersed in a medium (grey box). Numbers 1-5 indicate different levels of the two light beams ( $a$ and $b$ ) in the microscope.

a Sénarmont compensator, S, which consists of a quarter-wave plate, and finally pass through an analyzer, A.

We assume that the incident beam has one unit power of light intensity after passing through the polarizer $\mathrm{P}$ and that the beam splitter BS splits the incident beam into two rays (a and b; Figure 1) with equal intensity. The propagation of a polarized light beam can be treated with the Jones formalism, where the two components of the beam are given by a vector. When the beam passes through an optical element that changes its polarization, this is equivalent to a matrix operation. At level 1 (Figure 1), the two rays are then described by the Jones vectors: ${ }^{12}$

$$
\mathbf{E}_{\mathrm{a}}=\frac{1}{2}\left(\begin{array}{l}
1 \\
1
\end{array}\right) \quad \text { and } \quad \mathbf{E}_{\mathrm{b}}=\frac{1}{2}\left(\begin{array}{c}
1 \\
-1
\end{array}\right)
$$

When a light beam is monochromatic (wavelength $\lambda$ ), a plate with thickness $d$ and refractive index $n_{\mathrm{o}}$ causes a phase shift $\varphi_{\mathrm{o}}=k n_{\mathrm{o}} d$ ( $k=2 \pi / \lambda$ is the wavenumber). The reference medium, with (real) refractive index $n_{\mathrm{r}}$, that surrounds the object introduces a phase shift $\varphi_{\mathrm{r}}=k n_{\mathrm{r}} d$. Thus, the phase difference between the two rays at level 2 (Figure 1) is $\delta=k\left(n_{\mathrm{r}}-n_{\mathrm{o}}\right) d$. Generally, the refractive index of the object is complex, $n_{\mathrm{o}}=n_{\mathrm{oR}}+i n_{\mathrm{oI}}$, with real and imaginary parts $n_{\mathrm{oR}}$ and $n_{\mathrm{oI}}$. The phase shift created by the object is then also complex, or $\delta=\delta_{\mathrm{R}}+\mathrm{i} \delta_{\mathrm{I}}=\mathrm{k}\left(n_{\mathrm{r}}-n_{\mathrm{oR}}\right) d-i k n_{\mathrm{oI}} d$, i.e., $\delta_{\mathrm{R}}=k\left(n_{\mathrm{r}}-n_{\mathrm{oR}}\right) d$ and $\delta_{\mathrm{I}}=-k n_{\mathrm{oI}} d$.

The Jones matrix of the Sénarmont compensator, operating from level 3 to 4 (Figure 1), is ${ }^{12}$

$$
\mathbf{J}_{\mathrm{S}}=e^{i \pi / 4}\left(\begin{array}{cc}
1 & 0 \\
0 & -i
\end{array}\right)
$$

and the Jones matrix of the analyzer rotated over an angle $\alpha$ is

$$
\mathbf{J}_{\mathrm{A}}(\alpha)=\left(\begin{array}{cc}
\cos ^{2} \alpha & \sin \alpha \cos \alpha \\
\sin \alpha \cos \alpha & \sin ^{2} \alpha
\end{array}\right)
$$

Consequently, the Jones vector of the light beam at level 5 (Figure 1) is

$$
\mathbf{E}=\mathbf{J}_{\mathrm{A}}(\alpha) \mathbf{J}_{\mathrm{S}}\left(\mathbf{E}_{\mathrm{a}}+e^{-i \delta} \mathbf{E}_{\mathrm{b}}\right)=\frac{1}{2} e^{i(\pi / 4-\alpha)}\left[1+e^{i(2 \alpha-\delta)}\right]\left(\begin{array}{c}
\cos \alpha \\
\sin \alpha
\end{array}\right)
$$

The resulting light intensity of the beam

$$
I(\alpha)=\mathbf{E}^{*} \mathbf{E}=a \cos ^{2}(\alpha-\Delta \alpha)+b
$$

is a sinusoidal function of the analyzer angle $\alpha$ with period $\pi$ (or, $180^{\circ}$ ) and with amplitude $a=\exp \left(\delta_{\mathrm{I}}\right)$ and an offset $b=(1-a)^{2} / 4 ; \Delta \alpha$ is the phase difference between the reference and object intensity or the angle needed to rotate the analyzer to fully compensate for the phase 
difference induced by the object:

$$
\Delta \alpha=\delta_{\mathrm{R}} / 2=\pi\left(n_{\mathrm{r}}-n_{\mathrm{oR}}\right) d / \lambda
$$

(when the angle is given in degrees $\Delta \alpha=180\left(n_{\mathrm{r}}-n_{\mathrm{oR}}\right) d / \lambda$ ). The phase difference is a linear function of the reference fluid with slope $s=\pi d / \lambda$. From the slope, the thickness of the object can be derived: $d=s \lambda / \pi$.

The amplitude is directly related to the absorption coefficient, $\kappa$, of a homogeneous object because $\kappa=2 k n_{\mathrm{oI}}=-2 \delta_{\mathrm{I}} / d$, which can be derived from the transmittance, $T=\exp (-\kappa d)$, or

$$
a=\exp \left(-k n_{\mathrm{oI}} d\right)=\exp (-\kappa d / 2)=T^{1 / 2}
$$

When the imaginary part of the refractive index of the object is negligible, then $\delta$ is real; thus, $a=1$ and $b=0$. Then, $I(\alpha)=\cos ^{2}$ $(\alpha-\Delta \alpha)$, following the angle-dependent intensity function used for transparent objects (e.g. Ref. 8).

We note that the beam splitter BS and the beam combiner BC (Figure 1) are designed to be identical, and therefore, when the two beams $\mathrm{a}$ and $\mathrm{b}$ do not have the same amplitude, the formulae derived for the intensity and phase difference remain the same.

\section{Wing anatomy and pigmentation}

We have applied the above-described theory to the case of the American Rubyspot, Hetaerina americana, a damselfly of the family Calopterygidae. Most of the damselfly's wings are colorless and transparent, but they are strikingly red-colored near the thorax (Figure $2 \mathrm{a}$ and $2 \mathrm{~b}$ ). The wing is a meshwork of wing veins, forming a frame of cells with thin membranes (Figure 2 and $3 a$ ). In the red wing parts, both the veins and the membrane between the veins are red pigmented (Figure 2c). Scanning electron microscopy shows that the membranes approximate a thin film, but the surface is often rough due to numerous protrusions (Figure $3 \mathrm{~b}$ ). The membrane thickness

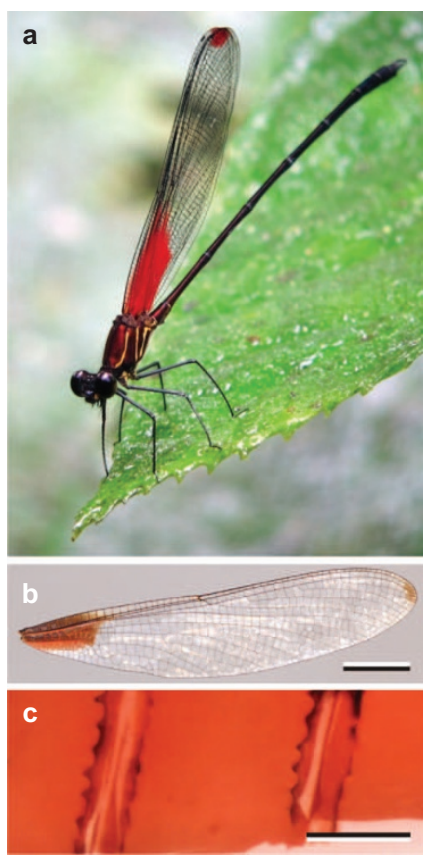

Figure 2 The American Rubyspot, Hetaerina americana. (a) Photograph (by Scott Olmstead) of a resting damselfly, showing the prominently red-pigmented wings. (b) A single wing, showing a meshwork of veins (bar=5 mm). (c) A small section of the red-pigmented wing part, showing that both the veins and the membrane in between are pigmented (bar $=0.1 \mathrm{~mm}$ ).
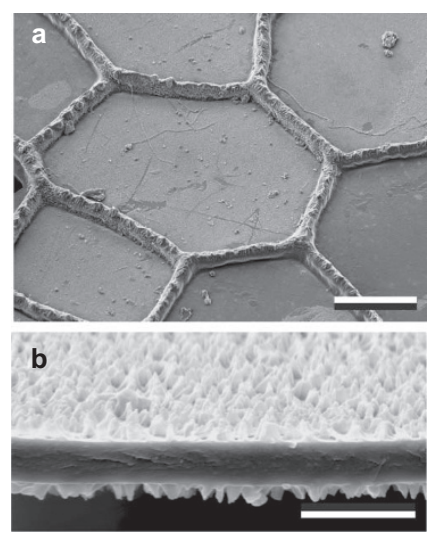

Figure 3 Scanning electron microscopy of a Hetaerina americana wing piece. (a) Wing veins surrounding a membrane window (bar $=100 \mu \mathrm{m})$. (b) A sectioned window membrane, showing numerous protrusions (bar $=5 \mu \mathrm{m}$ ).

varies from below $1 \mu \mathrm{m}$ to approximately $3 \mu \mathrm{m}$ from the wing tip to the base near the thorax.

The thin film properties of the membrane are immediately recognizable in the reflectance spectra (Figure 4). The reflectance spectrum
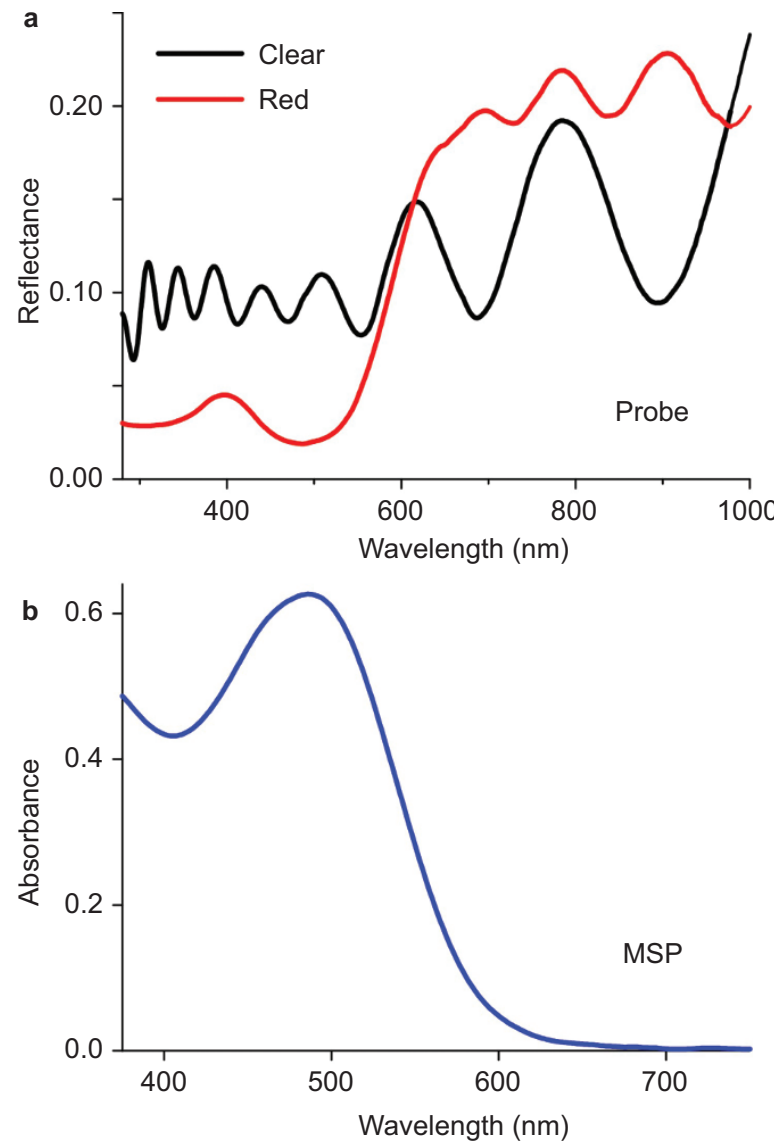

Figure 4 Wing reflectance and absorbance spectra of the American Rubyspot damselfly, Hetaerina americana. (a) Reflectance spectrum measured with a bifurcated fiber optic probe for clear, unpigmented, and red-pigmented wing pieces. (b) Absorbance spectrum measured by MSP for a window membrane from a section of the red-pigmented wing part, immersed in a fluid with a refractive index of 1.60. MSP, microspectrophotometry. 
of the unpigmented wing part (Figure 4a, clear) exhibits strong oscillations; the periodicity yields an average thickness of $0.87 \mu \mathrm{m}$. The reflectance measured in the red wing part (Figure 4a, red) is low in the short-wavelength region because the pigment has a peak absorption at $\sim 490 \mathrm{~nm}$ (Figure $4 \mathrm{~b}$ ). In the long-wavelength range, the reflectance spectrum shows oscillations corresponding to a thin film with varying thickness; the average thickness in the specific case of Figure $4 \mathrm{~b}$ was estimated to be $1.9 \mu \mathrm{m}$, but at other locations, the average thickness was different (in the range of $1-3 \mu \mathrm{m}$ ).

\section{Refractive index and absorption coefficient}

The effect of the absorbing pigment on the refractive index of the wing membranes can be studied with Jamin-Lebedeff interference microscopy. We therefore cut small sections out of the wing, immersed them in a fluid with a known refractive index, and investigated the sections via interference microscopy with monochromatic light. The image intensities of the wing section and the outer reference area vary sinusoidally with the angular position of the analyzer (Figure 5). Both the amplitude of the sinusoidal intensity of the wing section relative to the amplitude of the reference and the phase difference are wavelength-dependent (Figure 5d).

A series of measurements similar to those shown in Figure 5 were performed on three wing sections from the red wing parts, achieved by immersing the sections in immersion fluids with refractive indices of $1.51,1.55$ and 1.60 (at $586 \mathrm{~nm}$; Figure 6). The image intensity of an area in the wing section and that of a reference area outside the section were measured as a function of the analyzer position for a range of
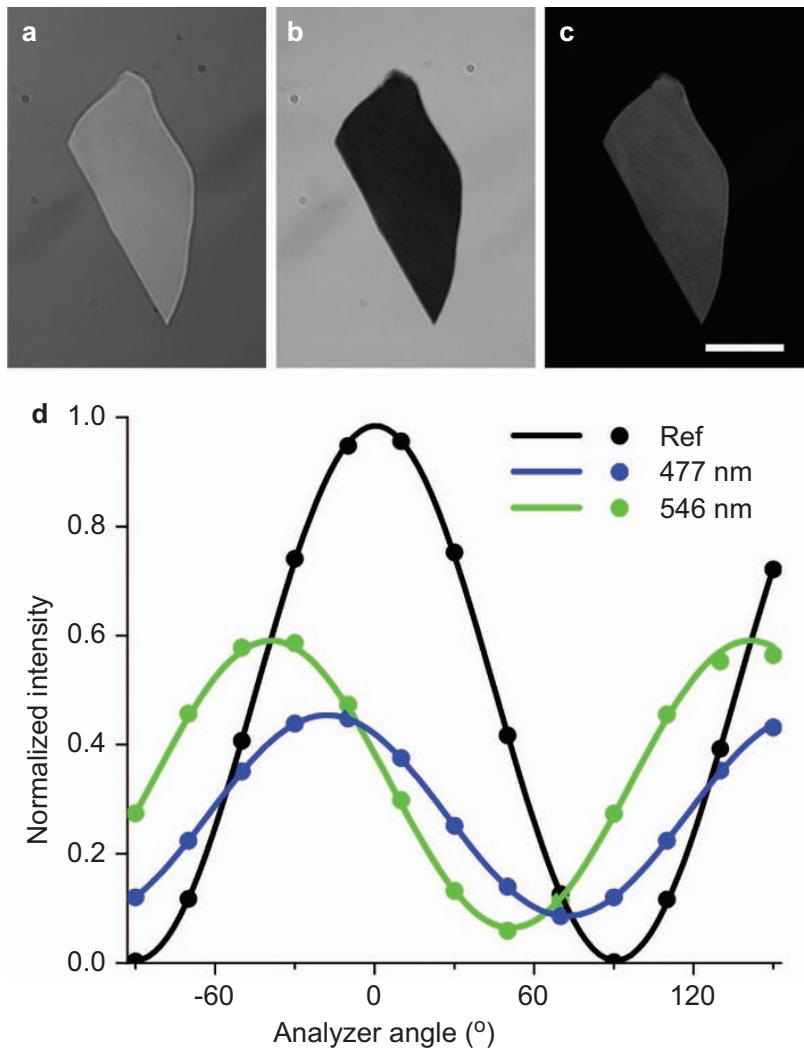

Figure 5 Jamin-Lebedeff microscopy of a wing piece of the damselfly $\mathrm{H}$. americana. (a-c) A piece of wing membrane immersed in a reference fluid with a refractive index of 1.51, observed with 546-nm illumination and an analyzer angle of $-50^{\circ}, 30^{\circ}$ and $110^{\circ}$ (bar $=10 \mu \mathrm{m}$ ). (d) Angular dependence of the image intensities of a reference area outside the wing piece (ref) and in the wing piece when illuminated with 477 and 546 nm light.
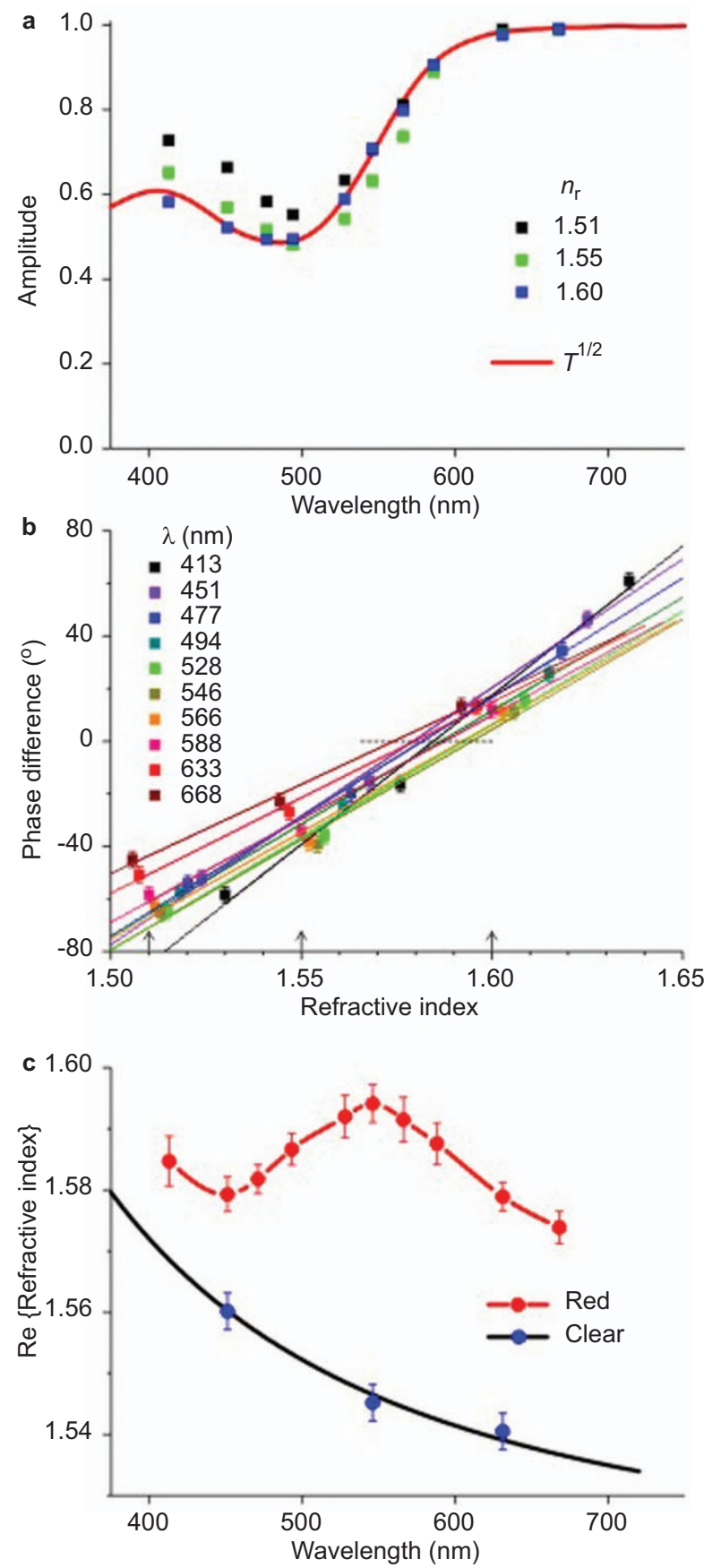

Figure 6 Amplitude and phase difference of the sinusoidal fits to the analyzer angle-dependent intensity curves measured for wing membrane pieces and the refractive index dispersion. (a) Amplitudes for three immersion fluids, with refractive index (at $589 \mathrm{~nm}$ ) $n_{\mathrm{r}}=1.51,1.55$ and 1.60 , together with $T^{1 / 2}$, the square root of the transmittance spectrum calculated from Figure $4 b$. (b) Phase difference as a function of the refractive index of the three reference fluids (values for $589 \mathrm{~nm}$ are indicated by arrows) for various wavelengths, $\lambda$, together with linear fits to the data for each wavelength. The real parts of the refractive index of the object and the reference are identical when the phase difference is zero (dashed horizontal line). (c) Real part of the refractive index of the wing membrane as a function of wavelength for the red-pigmented and the clear, unpigmented wing membrane (blue symbols). The black line shows the dispersion curve for insect chitin (from Ref. 8).

visible wavelengths. Figure 6a (symbols) shows the ratio of the amplitude of the resulting sinusoids calculated for each wavelength. According to Equation (7), the amplitude equals the square root of 
the transmittance. The absorbance is defined as $D(\lambda)=-\log _{10}[T(\lambda)]$, and the transmittance spectrum was thus calculated from the absorbance spectrum of Figure $4 \mathrm{~b}$ with $T(\lambda)=10^{-D(\lambda)}$ (Figure 6a, red line).

Figure $6 \mathrm{~b}$ shows the phase difference between the sinusoids of the wing sections and the reference as a function of wavelength for the three cases of immersion fluids. Linear functions fitted to the phase difference data points for each wavelength, as shown in Figure $6 \mathrm{~b}$, yielded a thickness of $d=2.5 \pm 0.1 \mu \mathrm{m}$. The fitted linear functions produced a zero value when the refractive index of the wing membrane equaled the refractive index of the reference: $n_{\mathrm{oR}}=n_{\mathrm{r}}$. This fitting yielded the refractive index spectrum of the wing membrane (Figure $6 \mathrm{c}$, red symbols and curve). The transparent wing area, which was also investigated at a few wavelengths, appeared to have a much lower refractive index (Figure 6c, blue symbols), coinciding well with the dispersion curve obtained for unpigmented chitin. ${ }^{8}$ This result suggests that the unpigmented wing regions consist of pure chitin and that the increase in the refractive index of the red-pigmented wing regions is caused by the presence of short-wavelength-absorbing pigment.

Figure $6 \mathrm{c}$ reveals that the refractive index of the red-pigmented wing membrane has a marked anomalous dispersion. A material generally exhibits anomalous dispersion in the wavelength range of a distinct absorption band. Therefore, it is likely that the measured dispersion curve (Figure $6 \mathrm{c}$ ) is caused by the absorbing pigment represented in Figure $4 \mathrm{~b}$. This assumption can be quantitatively assessed with the Kramers-Kronig dispersion relation (e.g., Refs. 13 and 14):

$$
\Delta n_{\mathrm{R} j}(\lambda)=\frac{1}{2 \pi^{2}} \int_{0}^{\infty} \frac{\kappa_{j}\left(\lambda^{\prime}\right)}{1-\left(\lambda^{\prime} / \lambda\right)^{2}} \mathrm{~d} \lambda^{\prime}
$$

which yields the contribution to the real part of the refractive index, $\Delta n_{\mathrm{R} j}$, from band $j$ of the absorption spectrum of the pigment with absorption coefficient $\kappa_{j}(\lambda)$. The absorption coefficient of the wing membrane medium can be derived from the transmittance spectrum, $T(\lambda)$, and from the absorbance spectrum, $D(\lambda)$, with $\kappa(\lambda)=-\ln [T(\lambda)] / d=2.3 D(\lambda) / d$. Using the estimated thickness $d=2.5 \mu \mathrm{m}$, the absorption coefficient spectrum of Figure $7 \mathrm{a}$ (red curve) was obtained. To allow for proper Kramers-Kronig calculations, the spectrum was extrapolated to the shorter wavelength range and was assumed to consist of an $\alpha$ and $\beta$ band, which were fitted with modified lognormal functions (see the section on 'Materials and methods'; Figure 7b, blue curve). Figure 7b shows the contributions to the refractive index calculated from Equation (8). Both absorption bands create a distinct anomalous dispersion curve; for the $\alpha$ band, a peak at $\sim 550 \mathrm{~nm}$ and a valley at $\sim 430 \mathrm{~nm}$ are obtained. The $\beta$ band increases the refractive index in the visible wavelength range, where it exhibits normal dispersion, reducing the modulation in the anomalous dispersion curve of the $\alpha$ band. Figure $7 \mathrm{~b}$ furthermore shows the difference between the refractive indices of the damselfly's red and clear wing membranes (red curve), as calculated from Figure 6c. The latter spectrum is shifted upwards from the calculated dispersion curve by about $0.2-0.3$. An obvious interpretation of this upward shift is that it is caused by absorbing bands located at wavelengths of less than $300 \mathrm{~nm}$. These bands could, for instance, arise from specific proteins to which the pigment molecules are bound and that have strong absorption bands in the very short-wavelength range.

The nature of the pigment of the damselfly wings is uncertain, but the absorption spectrum resembles that of xanthommatin, ${ }^{15}$ a member of the ommochromes, a family of pigments widely encountered among insects. ${ }^{16}$ The pigment density is high, with a peak absorption coefficient of $\sim 0.5 \mu \mathrm{m}^{-1}$, resulting in a substantial anomalous dispersion
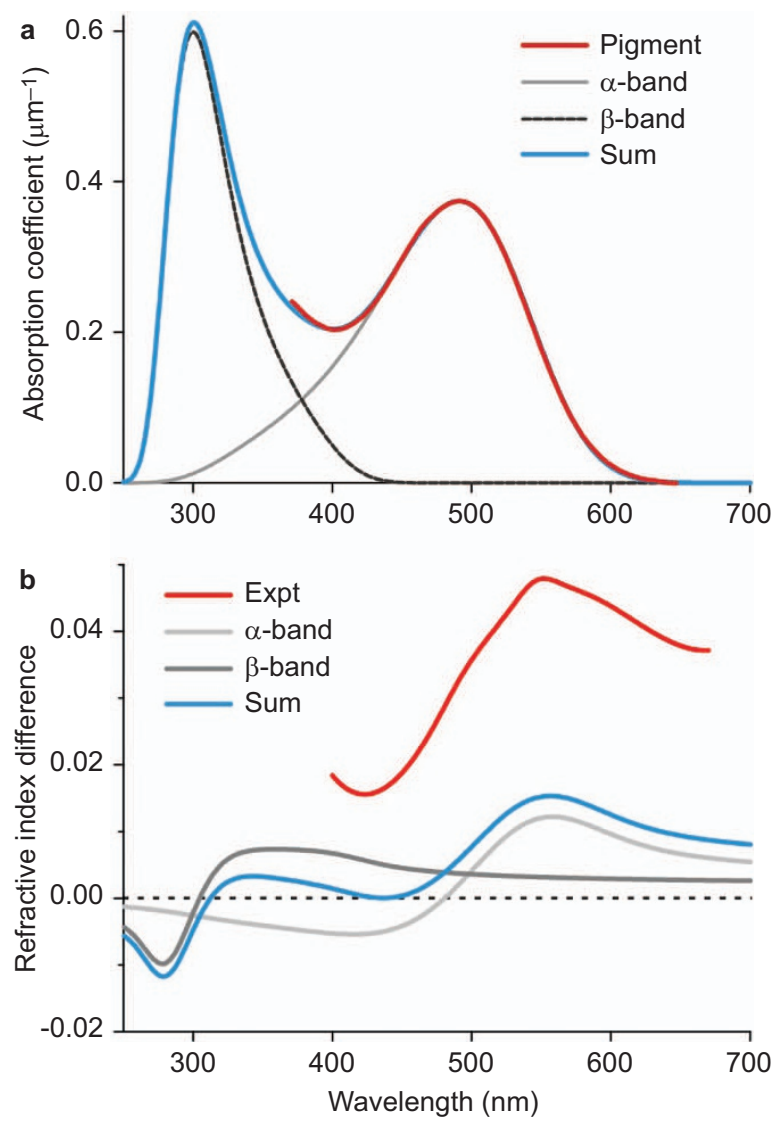

Figure 7 Absorption coefficient of the pigment in the wings of Hetaerina americana and the resulting contribution to the refractive index. (a) The absorption coefficient spectrum derived from the absorbance spectrum of Figure $4 \mathrm{~b}$ with a membrane thickness of $2.5 \mu \mathrm{m}$. The spectrum is decomposed into two components, an $\alpha$ band and a $\beta$ band, which are assumed to follow modified lognormal functions (see the section on 'Materials and methods'). (b) The difference between the refractive indices of the membranes in the red and clear wing parts derived from Figure $6 \mathrm{c}$ (expt) and the contributions to the refractive index from the components of the absorption coefficient spectrum (the $\alpha$ and $\beta$ bands and their sum) calculated with the Kramers-Kronig dispersion relation (Equation (8)).

of the refractive index, with a peak value of $\sim 1.595$ at $550 \mathrm{~nm}$ and a low of $\sim 1.578$ at $450 \mathrm{~nm}$ (Figure $6 \mathrm{c}$ ). The local density of the pigment shows some variation and thus results in a variable transmittance. This feature presumably causes the slightly variable wavelength dependence of the sinusoid amplitudes (Figure $5 c$ ). The wing membranes are not ideal thin films due to numerous protrusions, which create a rough surface (for similar rough membrane surfaces on dragonfly wings, see Ref. 17). The protrusions are expected to have a negligible effect on the measurements because of the small difference between the refractive indices of the wing membranes and the reference fluid.

Generally, in pigmentary coloration, a short-wavelength-absorbing pigment reduces the reflection/scattering of incident light in the shortwavelength range, leaving the longer wavelength light relatively unobstructed, thus resulting in a yellow, orange or red color, depending on the extent of the pigment's absorption spectrum. Interestingly, a high pigment density causes an increase in the real part of the refractive index in the longer wavelength range, which will enhance the reflectivity of the tissue at the longer wavelengths. Preliminary measurements in several butterfly species suggest that this optical effect of enhancing the color contrast by increasing the refractive index is 
widespread. The dual effect of pigment absorption, namely, the absorption of short-wavelength light and the enhanced reflection of long-wavelength light, can also be recognized in reflecting pigment cells and tapeta in the eyes of crustaceans ${ }^{18-20}$ and presumably also in the coloration caused by pigments in cephalopods. ${ }^{21}$

We previously studied the pigmented wings of another damselfly, Calopteryx japonica. ${ }^{22}$ The pigment in that case is melanin, causing brown-colored wings in the immature female, the mature female and the immature male. However, the mature males have distinctly bluecolored wings. The blue color is present in the wing veins, where melanin is concentrated in layers that alternate with relatively unpigmented layers, thus creating an optical multilayer that strongly reflects in the blue wavelength range. The melanin pigment in this case causes structural coloration. The refractive index dispersion of the melanin layers was found to be normal in the visible wavelength range, that is, the refractive index decreases with increasing wavelength, due to the very broad melanin absorption spectrum, which decreases monotonically with increasing wavelength from the ultraviolet to the infrared region. The strong absorption of the melanin in the very short-wavelength range causes the melanin to endow the tissue with a very high real part of the refractive index, resulting in local refractive index values above $1.8 .^{22}$ Consequently, when the melanin is periodically arranged, strong photonic effects can emerge. This possibility is exploited by many animals, e.g., in bird feathers ${ }^{23-25}$ and beetle cuticles ${ }^{26,27}$ (see also Refs. 28 and 29). We note that there are even several examples in which periodically arranged, structurally colored tissue contains additional pigment that selectively filters the reflected light, thus tuning and sharpening the reflectance spectrum. ${ }^{21,30,31}$ Pigmentary and structural coloration thus are not strictly separable.

\section{CONCLUSION}

Using a newly developed method based on Jamin-Lebedeff interference microscopy, we have investigated the red-pigmented wings of the damselfly $H$. americana and quantitatively assessed the contribution of the blue-absorbing pigment to the overall refractive index. In all cases of animal coloration, the refractive index of the colored tissue plays a central role, specifically in the very thin tissues of insect wings, butterfly wing scales and bird feather barbules, which often display a strongly saturated pigmentary color. The latter example implies a high pigment density with high refractive indexes as an immediate consequence. As the refractive index depends on wavelength, i.e., featuring dispersion, a quantitative knowledge of the refractive index is key to understanding the resulting coloration. The novel approach based on Jamin-Lebedeff interference microscopy for absorbing media, as outlined in the present paper, allows for straightforward measurements of the refractive index of microscopic objects, which will be valuable for gaining quantitative insight into how the material properties of animal tissues influence coloration.

\section{ACKNOWLEDGMENTS}

We thank Dr D Goldstein and Dr P Vukusic for their comments and Professor J Th de Hosson for the use of electron microscope facilities. This study was financially supported by the Air Force Office of Scientific Research/European Office of Aerospace Research and Development AFOSR/EOARD (grant FA8655-08-1-3012).

1 Saks L, McGraw KJ, Hõrak P. How feather colour reflects its carotenoid content. Funct Ecol 2003; 17: 555-561.
2 Shawkey MD, Hill GE. Carotenoids need structural colours to shine. Biol Lett 2005; 1 : 121-124.

3 Wijnen B, Leertouwer HL, Stavenga DG. Colors and pterin pigmentation of pierid butterfly wings. J Insect Physiol 2007; 53: 1206-1217.

4 Vukusic P, Stavenga DG. Physical methods for investigating structural colours in biological systems. J R Soc Interface 2009; 6(Suppl 2): S133-S148.

5 Gillis JM, Wibo M. Accurate measurement of the thickness of ultrathin sections by interference microscopy. J Cell Biol 1971; 49: 947-949.

6 Schäfer FJ, Kleemann W. High precision refractive index measurements revealing order parameter fluctuations in $\mathrm{KMnF}_{3}$ and NiO. J Appl Phys 1985; 57: 2606-2612.

7 Stavenga DG, Kruizinga R, Leertouwer HL. Dioptrics of the facet lenses of male blowflies Calliphora and Chrysomia. J Comp Physiol A 1990; 166: 365-371.

8 Leertouwer HL, Wilts BD, Stavenga DG. Refractive index and dispersion of butterfly scale chitin and bird feather keratin measured by interference microscopy. Opt Express 2011; 19: 24061-24066.

9 Stavenga DG, Tinbergen J, Leertouwer HL, Wilts BD. Kingfisher feathers-colouration by pigments, spongy nanostructures and thin films. J Exp Biol 2001; 214: 3960-3967.

10 Stavenga DG, Smits RP, Hoenders BJ. Simple exponential functions describing the absorbance bands of visual pigment spectra. Vision Res 1993; 33: 1011-1017.

11 Schneider CA, Rasband WS, Eliceiri KW. NIH Image to ImageJ: 25 years of image analysis. Nat Methods 2012; 9: 671-675.

12 Goldstein D. Polarized Light. New York/Basel: Marcel Dekker; 2003.

13 Bell EE. Optical constants and their measurements. In: Genzel L, editor. Handbuch der Physik. Vol. XXV/2a. Berlin: Springer; 1967. pp1-58.

14 Stavenga DG, van Barneveld HH. On dispersion in visual photoreceptors. Vision Res 1975; 15: 1091-1095.

15 Nijhout HF. Ommochrome pigmentation of the linea and rosa seasonal forms of Precis coenia (Lepidoptera: Nymphalidae). Arch Insect Biochem Physiol 1997; 36: 215-222.

16 Reed RD, Nagy LM. Evolutionary redeployment of a biosynthetic module: expression of eye pigment genes vermilion, cinnabar, and white in butterfly wing development. Evol Dev 2005; 7: 301-311.

17 Hooper IR, Vukusic P, Wootton RJ. Detailed optical study of the transparent wing membranes of the dragonfly Aeshna cyanea. Opt Express 2006; 14: 4891-4897.

18 Exner S. Die Physiologie der facittirten Augen von Krebsen und Insecten. Leipzig: Deuticke; 1891.

19 Exner S. The Physiology of the Compound Eyes of Insects and Crustaceans (translated by R.C. Hardie). Berlin/Heidelberg: Springer; 1989.

20 Doughtie DG, Rao KR. Ultrastructure of the eyes of the grass shrimp, Palaemonetes pugio. Cell Tiss Res 1984; 238: 271-288.

21 Mäthger LM, Hanlon RT. Malleable skin coloration in cephalopods: selective reflectance, transmission and absorbance of light by chromatophores and iridophores. Cell Tiss Res 2007; 329: 179-186.

22 Stavenga DG, Leertouwer HL, Hariyama T, de Raedt HA, Wilts BD. Sexual dichromatism of the damselfly Calopteryx japonica caused by a melanin-chitin multilayer in the male wing veins. PLOS ONE 2012; 7: e49743.

23 Durrer H. Schillerfarben der Vogelfeder als Evolutionsproblem. Denkschr Schweiz Naturforsch Ges 1977; 91: 1-126.

24 Stavenga DG, Leertouwer HL, Marshall NJ, Osorio D. Dramatic colour changes in a bird of paradise caused by uniquely structured breast feather barbules. Proc $R$ Soc B2010; 278: 2098-2104.

25 Kinoshita S, Yoshioka S, Miyazaki J. Physics of structural colors. Rep Prog Phys 2008; 71: 076401.

26 Hariyama T, Hironaka M, Takaku Y, Horiguchi H, Stavenga DG. The leaf beetle, the jewel beetle, and the damselfly; insects with a multilayered show case. In: Kinoshita S, Yoshioka S, editors. Structural Color in Biological Systems-Principles and Applications. Osaka: Osaka University Press; 2005. pp153-176.

27 Stavenga DG, Wilts BD, Leertouwer HL, Hariyama T. Polarized iridescence of the multilayered elytra of the Japanese Jewel Beetle, Chrysochroa fulgidissima. Phil Trans R Soc B 2011; 366: 709-723.

28 Land MF. The physics and biology of animal reflectors. Prog Biophys Mol Biol 1972; 24: 77-105.

29 Kinoshita S. Structural Colors in the Realm Of Nature. Singapore: World Scientific: 2008.

30 Wilts BD, Michielsen K, de Raedt H, Stavenga DG. Iridescence and spectral filtering of the gyroid-type photonic crystals in Parides sesostris wing scales. Interface Focus 2012; 2: 681-687.

31 Trzeciak TM, Wilts BD, Stavenga DG, Vukusic P. Variable multilayer reflection together with long-pass filtering pigment determines the wing coloration of papilionid butterflies of the nireus group. Opt Express 2012; 20: 8877-8890.

This work is licensed under a Creative Commons AttributionNonCommercial-NoDerivs Works 3.0 Unported license. To view a copy of this license, visit http://creativecommons.org/licenses/by-nc-nd/3.0 